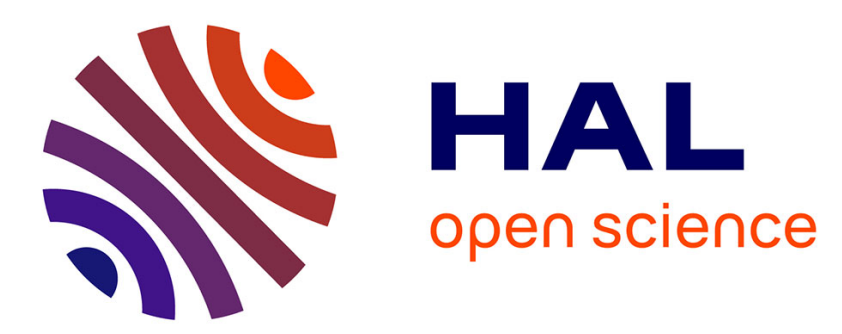

\title{
Possible M1 - E1 interference effects in m1 forbidden transitions with crossed magnetic and electric fields
}

\author{
M.A. Bouchiat, M. Poirier
}

\section{To cite this version:}

M.A. Bouchiat, M. Poirier. Possible M1 - E1 interference effects in $\mathrm{m} 1$ forbidden transitions with crossed magnetic and electric fields. Journal de Physique, 1982, 43 (5), pp.729-736. 10.1051/jphys:01982004305072900 . jpa-00209445

\section{HAL Id: jpa-00209445 https://hal.science/jpa-00209445}

Submitted on 1 Jan 1982

HAL is a multi-disciplinary open access archive for the deposit and dissemination of scientific research documents, whether they are published or not. The documents may come from teaching and research institutions in France or abroad, or from public or private research centers.
L'archive ouverte pluridisciplinaire HAL, est destinée au dépôt et à la diffusion de documents scientifiques de niveau recherche, publiés ou non, émanant des établissements d'enseignement et de recherche français ou étrangers, des laboratoires publics ou privés. 


\title{
Possible $M_{1}-E_{1}$ interference effects in $M_{1}$ forbidden transitions with crossed magnetic and electric fields
}

\author{
M. A. Bouchiat and M. Poirier ${\left({ }^{*}\right)}^{*}$ \\ Laboratoire de Spectroscopie Hertzienne (**), Ecole Normale Supérieure, \\ 24, rue Lhomond, 75231 Paris Cedex 05, France
}

(Reçu le 13 novembre 1981, accepté le 15 janvier 1982)

\begin{abstract}
Résumé. - Nous prédisons dans ce papier de nouveaux effets d'interférence possibles, $M_{1}-E_{1}$ Stark, qui doivent apparaître dans les transitions interdites $n \mathrm{~S}-n^{\prime} \mathrm{S}$, si l'on applique un champ magnétique statique croisé avec le champ électrique statique. En particulier, il devient possible de mesurer le rapport $M_{1} / E_{1}$ Stark directement sur l'intensité de fluorescence. Ces effets sont importants aussi en ce qui concerne le problème des erreurs systématiques dans les expériences de violation de la parité et donne de nouveaux moyens de calibration du signal cherché.
\end{abstract}

\begin{abstract}
In this paper we predict new possible $M_{1}-E_{1}$ Stark interference effects which should appear in $n S-n^{\prime} S M_{1}$ forbidden transitions when a static magnetic field crossed with the static electric field is applied. In particular it becomes possible to measure the ratio $M_{1} / E_{1}$ Stark directly on the fluorescence intensity. These effects are also of importance for the problem of systematic errors in Parity Violation experiments and give new ways of calibrating the PV signal.
\end{abstract}

Introduction. - Several experiments involving interference between Stark induced electric dipole amplitude $\left(E_{1}^{\text {ind }}\right)$ and magnetic dipole amplitude $\left(M_{1}\right)$ in radiative forbidden $M_{1}$ transitions have developed in the last few years, in connection with actual problems in Atomic Physics. Firstly, one can determine by this way the magnitude and the sign of very weak $M_{1}$ amplitudes [1]. Such measurements have already been performed on cesium [2], on thallium. [3] and on methane [4]. Extension to hydrogen-like and heliumlike atoms may result in a test of QED calculations, and is presently under active investigation [5]. Secondly, the $M_{1} E_{1}^{\text {ind }}$ interference occurs in parity violation experiments, like those being performed at present on cesium [6] and thallium [7] in zero magnetic field and also those under investigation on the same elements with the use of a high magnetic field $[8,9]$.

However, up to now, the $M_{1} E_{1}^{\text {ind }}$ interference effects have only been observed in zero magnetic field. In this case, they can only appear in the electronic polarization $\mathbf{P}_{\mathrm{e}}$ of the excited state, and not in its global population. Besides, they can be observed with

(*) Present address : SPAS, C.E.N. Saclay, 91191 Gif sur Yvette, France.

$\left({ }^{* *}\right)$ Laboratoire associé au C.N.R.S. rectilinear as well as circular incident polarization, and in this last case they do not depend on the photon helicity. Here we show that a static magnetic field greatly modifies these features, because of the new symmetry acquired by the atomic wave function. For instance, in a magnetic field, an helicity dependence can appear on the $E_{1}^{\text {ind }} M_{1}$ interference. More generally, it can be shown that a magnetic field along a circularly polarized laser beam induces circular dichroism and thus stray d.c. magnetic fields can mimic parity violation in zero magnetic field experiments. Consequently, we develop a systematic calculation of spurious signals due to a weak longitudinal magnetic field in the cesium experiment.

On the other hand, a magnetic field as large as several hundred gauss can generate new $E_{1}^{\text {ind }} M_{1}$ interference effects on the populations of the excited state, i.e. on the total fluorescence rate. This provides us with a measurement method of $M_{1}$ without need of polarization detection. A special attention will be paid to a contribution to $M_{1}$ (hereafter called the hyperfine magnetic dipole amplitude $M_{1}^{\text {hf }}$ ) which originates in the hyperfine mixing of the two S states. Lastly, we analyse the effect of the motional electric field responsible for an interference effect behaving exactly like $E_{1}^{\text {ind }} M_{1}$ : it can affect the measurement of $M_{1}$ in a magnetic field, so it is included in our calculation. 
Section 1 is devoted to symmetry arguments showing which geometrical configuration is required to observe $E_{1}^{\text {ind }} M_{1}$ interference effects without and with a magnetic field. The new features of $H$ dependent effects are emphasized. In section 2, we develop our mathematical formalism. In section 3, we present the result of the calculation of the left-right asymmetry due to a stray longitudinal $H$ field. The conditions in which such a signal can simulate an asymmetry characteristic of parity violation are discussed. At last, section 4 deals with the $E_{1}^{\text {ind }}-M_{1}$ interference effects in the presence of a large transverse magnetic field.

1. Conditions for an $E_{1}^{\text {ind }} M_{1}$ interference. - Simple symmetry considerations will be the starting point of our search for $E_{1}^{\text {ind }} M_{1}$ interference effects.

In the Cs experiment, one detects the stationary component of the total or circularly polarized fluorescence rate from $n^{\prime} \mathrm{S}_{1 / 2}$ to $n^{\prime \prime} \mathrm{P}_{1 / 2}$, namely $7 \mathrm{~S}_{1 / 2}$ to $6 \mathrm{P}_{1 / 2}$. The total fluorescence rate is a scalar which must be expressed in terms of the experimental parameters : the applied static fields, electric $\left(\mathbf{E}_{0}\right)$ and magnetic $(\mathbf{H})$, the polarization $(\boldsymbol{\varepsilon})$ and linear momentum $\left(\mathbf{k}_{\mathbf{i}}\right)$ of the laser beam; if the polarization is circular, we replace $\varepsilon$ by the longitudinal angular momentum of the photon defined as $i \varepsilon \times \varepsilon^{*}=\xi_{\mathrm{i}} \hat{k}_{\mathrm{i}}$ $\left(\hat{k}_{\mathrm{i}}\right.$ stands for a unit vector along $\mathbf{k}_{\mathrm{i}}, \boldsymbol{\varepsilon}$ is a complex vector of unit norm). In a similar way, the electronic polarization (responsible for the polarized fluorescence rate) is an axial vector which must be expressed in terms of the quantities $\mathbf{E}_{0}, \mathbf{H}, \mathbf{k}_{\mathrm{i}}$ and $\boldsymbol{\varepsilon}$ (or $\xi_{\mathrm{i}} \hat{k}_{\mathrm{i}}$ ). The $E_{1}^{\text {ind }} M_{1}$ interference effects can be investigated with the help of the following criterion : first, they are purely electromagnetic phenomena which thus preserve the invariance under parity $P$ and time reversal $T$. Then, they are of course linear against $\mathbf{E}_{0}$, against $\mathbf{k}_{\mathrm{i}}$ (appearing in $M_{1}$ ) and quadratic in $\varepsilon$ (since each transition amplitude is itself proportional to $\varepsilon$ ). Without a magnetic field, it results from the symmetry properties of $\mathbf{E}_{0}, \mathbf{k}_{\mathrm{i}}, \boldsymbol{\varepsilon}$ and $\xi_{\mathrm{i}}$ that no $P_{+} T_{+}$ scalar with the afore mentioned properties can be found. This means that no $E_{1}^{\text {ind }} M_{1}$ interference occurs on the total fluorescence rate for $H=0$. Besides, the only allowed expressions for the atomic orientation $\mathbf{P}_{\mathrm{e}}$, which must be an axial vector, odd under $T$, are : $(\boldsymbol{\varepsilon} . \varepsilon)\left(\mathbf{k}_{\mathrm{i}} \times \mathbf{E}_{0}\right),\left(\mathbf{E}_{0} \cdot \varepsilon\right)\left(\mathbf{k}_{\mathrm{i}} \times \boldsymbol{\varepsilon}\right)$ or $\left(\mathbf{E}_{0} \times \varepsilon . \mathbf{k}_{\mathrm{i}}\right) \varepsilon$ for a linear incident polarization and only $\mathbf{k}_{\mathrm{i}} \times \mathbf{E}_{0}$ for a circular incident polarization. In this expression, the photon helicity does not appear. The symmetry conditions exclude the possibility of a $\xi_{i}$ dependence. In fact, the existence of a $\mathbf{P}_{e}$ component linear in $\xi_{\mathrm{i}}$ and $\mathbf{E}_{0}$ for $H=0$ means parity violation [1] : in the PV experiment in zero $H$ field performed on Cs [6] and $\mathrm{Tl}$ [7], the searched for effect involves the existence in $\mathbf{P}_{\mathrm{e}}$ of the true vector quantity $\xi_{\mathrm{i}} \mathbf{E}_{0} \times \mathbf{k}_{\mathrm{i}}$.

This is no longer true with a non zero $H$ field. We expect that a stray magnetic field can simulate the parity violation effect by creating a new $E_{1}^{\text {ind }} M_{1}$ interference linear in $\xi_{i}$ and $\mathbf{E}_{0}$ which contributes to $\mathbf{P}_{\mathrm{e}}$. Indeed, if we try to form an axial vector, odd under time reversal (as the polarization $\mathbf{P}_{\mathrm{e}}$ must be), and linear against $\mathbf{E}_{0}, \xi_{\mathrm{i}} \mathbf{k}_{\mathrm{i}}$ and $\mathbf{H}$ (since we are looking for first order effects in the magnetic field), we find :

$$
\mathbf{P}_{\mathrm{e}} \propto\left(\xi_{\mathrm{i}} \mathbf{k}_{\mathrm{i}} \cdot \mathbf{H}\right)\left(\mathbf{k}_{\mathrm{i}} \times \mathbf{E}_{0}\right) \text { or } \mathbf{P}_{\mathrm{e}} \propto \xi_{\mathrm{i}} \mathbf{H} \times \mathbf{E}_{0} .
$$

If in the PV experiment, $\mathbf{E}_{0}, \mathbf{k}_{\mathrm{i}}$ and the observation direction $\mathbf{k}_{\mathrm{f}}$ are orthogonal, we can conclude that only a magnetic field along the laser beam can create an electronic polarization simulating parity violation, i.e. directed along $\mathbf{k}_{\mathbf{i}} \times \mathbf{E}_{0}$ and linear against $\xi_{\mathrm{i}}$ and $\mathbf{E}_{0}$. A perturbative calculation of this $E_{1}^{\text {ind }} M_{1}$ interference effect is described in section 3 .

Moreover, a static magnetic field can generate an $E_{1}^{\text {ind }} M_{1}$ interference on the total fluorescence rate. Restricting the discussion to linear incident polarization, let us write the list of $P_{+} T_{+}$scalars which are linear functions of $\mathbf{E}_{0}, \mathbf{k}_{\mathbf{i}}$ and $\mathbf{H}$. Noting that $\left(\mathbf{k}_{\mathbf{i}} \times \mathbf{E}_{\mathbf{0}} \cdot \mathbf{H}\right)$ has the right symmetry and inserting twice the wave polarization $\varepsilon$ as we should, we get the following candidates $\left({ }^{1}\right)$ :

$$
\left(\mathbf{E}_{0} \cdot \varepsilon\right)\left(\mathbf{H} . \mathbf{k}_{\mathrm{i}} \times \varepsilon\right) \text { and }\left(\mathbf{E}_{0} \cdot \mathbf{k}_{\mathrm{i}} \times \varepsilon\right)(\mathbf{H} . \varepsilon) \text {. }
$$

In order to make those quantities maximal, $\mathbf{E}_{0}, \mathbf{H}$ and $\mathbf{k}_{\mathrm{i}}$ have to be oriented along the three axes of a rectangular coordinate system, and $\varepsilon$ must be parallel either to $\mathbf{E}_{0}$ or to $\mathbf{H}$ as represented on figure $1 a$ and figure $1 b$ respectively. These configurations will be studied in section 4. On the contrary, in high-field PV experiments, one may search to minimize the importance of this effect and this is achieved by choosing $\mathbf{E}_{0}$ and $\mathbf{k}_{\mathrm{i}}$ parallel [8].

Before closing this section, we would like to make a remark concerning the validity of the criterion used to insure time reversal invariance in $E_{1}^{\text {ind }} M_{1}$ interference effects. Our considerations are valid only for stationary states [10] and strictly speaking do not apply to an unstable state, like the Cs $7 \mathrm{~S}$ state. However, in all the physical quantities we shall consider, the natural lifetime will not appear explicitly, so these quantities behave under time reversal as if
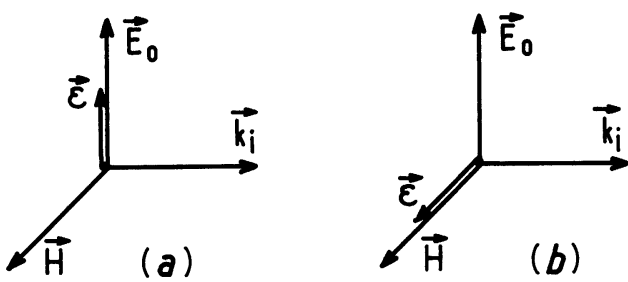

Fig. 1. - Optimal configurations for observing an $E_{1}^{\text {ind }} M_{1}$ interference on the total fluorescence rate.

$\left({ }^{1}\right)$ It can be shown that there is actually no other invariant which can satisfy all the required conditions. Note also that even if we relax the condition of linearity in $H$, the most general invariant is of the form $\left(\mathbf{k}_{\mathrm{i}} \times \mathbf{E}_{0} . \mathbf{H}\right) f\left(H^{2}\right)$ which just represents the same effect to higher orders in $\mathbf{H}$. 
the $7 \mathrm{~S}$ state were a stable state (see section 2). A very different situation occurs for the experiments completed on hydrogen atoms in the $2 S_{1 / 2}$ state in a magnetic field close to the crossing of the metastable and the highly damped $2 \mathrm{P}_{1 / 2}$ state [11].

\section{Physical parameters and mathematical forma-} lism. - The treatment given in this section applies directly to the case of an alkali $n S_{1 / 2} \rightarrow n^{\prime} \mathrm{S}_{1 / 2}$ transition excited by a nearly resonant laser beam. We recall that the fluorescence signal observed through a circular analyser transmitting the photons emitted in the direction $\hat{k}_{\mathrm{f}}$ with helicity $\xi_{\mathrm{f}}$ on the allowed $n^{\prime} \mathrm{S}_{1 / 2} \rightarrow n^{\prime \prime} \mathrm{P}_{1 / 2}$ transition, can be written :

$$
S=\frac{J}{2}\left(1+\xi_{\mathrm{f}} \mathbf{P}_{\mathrm{e}} \cdot \hat{k}_{\mathrm{f}}\right)
$$

where $J$ is the total fluorescence rate and $\mathbf{P}_{\mathrm{e}}$ the electronic polarization in the upper state. We wish to express $\mathfrak{J}$ and $\mathfrak{J} \mathbf{P}_{\mathrm{e}}$ in terms of populations and coherences of the upper state. The atomic states perturbed by the magnetic field are denoted $|n S \varepsilon M\rangle$ (ground state) and $\left|n^{\prime} S \varepsilon^{\prime} M^{\prime}\right\rangle$ (excited state), $\varepsilon= \pm 1$ (resp. $\varepsilon^{\prime}$ ) being defined in the following way : when the magnetic field tends to zero, by continuity $|\varepsilon, M\rangle$ tends to $\left|F=I+\frac{\varepsilon}{2}, M\right\rangle\left(\right.$ and $\left|\varepsilon^{\prime}, M^{\prime}\right\rangle$ to $\left|F^{\prime}=I+\frac{\varepsilon^{\prime}}{2}, M^{\prime}\right\rangle, I$ being the nuclear spin $F\left(F^{\prime}\right)$ the total angular momentum and $M\left(M^{\prime}\right)$ the magnetic quantum number. If $\rho_{n^{\prime}}$ is the density matrix restricted to the upper level, we can write, except for a proportionality coefficient, and neglecting the hyperfine coherences :

$$
\begin{aligned}
\mathfrak{J} & =\sum_{\varepsilon^{\prime} M^{\prime}}\left\langle\varepsilon^{\prime} M^{\prime}\left|\rho_{n^{\prime}}\right| \varepsilon^{\prime} M^{\prime}\right\rangle=\operatorname{Tr} \rho_{n^{\prime}} \\
\mathfrak{J} \mathbf{P}_{\mathrm{e}} & =\sum_{\varepsilon^{\prime} M_{1}^{\prime} M_{2}^{\prime}}\left\langle\varepsilon^{\prime} M_{1}^{\prime}\left|\rho_{n^{\prime}}\right| \varepsilon^{\prime} M_{2}^{\prime}\right\rangle\left\langle\varepsilon^{\prime} M_{2}^{\prime}|\boldsymbol{\sigma}| \varepsilon^{\prime} M_{1}^{\prime}\right\rangle=\operatorname{Tr} \rho_{n^{\prime}} \boldsymbol{\sigma} .
\end{aligned}
$$

The calculation of the matrix elements of $\rho_{n^{\prime}}$ is rather simple : owing to the very high suppression factor of the transition, the stimulated emission process can be totally ignored. This calculation is presented in the appendix. A perturbative treatment up to the first order in the laser intensity leads to :

$$
\begin{aligned}
\left\langle\varepsilon_{1}^{\prime} M_{1}^{\prime}\left|\rho_{n^{\prime}}\right| \varepsilon_{2}^{\prime} M_{2}^{\prime}\right\rangle=\frac{|\varepsilon|^{2}}{\hbar^{2}} \frac{1}{2(2 I+1)}\left(\omega_{n^{\prime} \varepsilon_{1}^{\prime} M_{1}^{\prime}, n^{\prime} \varepsilon_{2}^{\prime} M_{2}^{\prime}}-i \Gamma_{n^{\prime}}\right)^{-1} \times \sum_{\varepsilon M}\left\langle\varepsilon_{1}^{\prime} M_{1}^{\prime}|\tau| \varepsilon M\right\rangle\left\langle\varepsilon M\left|\tau^{+}\right| \varepsilon_{2}^{\prime} M_{2}^{\prime}\right\rangle \times \\
\quad \times\left(\frac{1}{\omega_{\mathrm{r}}-\omega_{n^{\prime} \varepsilon_{1}^{\prime} M_{1}^{\prime}, n \varepsilon M}+i \frac{\Gamma_{n^{\prime}}}{2}}-\frac{1}{\omega_{\mathrm{r}}-\omega_{n^{\prime} \varepsilon_{2}^{\prime} M_{2}^{\prime}, n \varepsilon M}-i \frac{\Gamma_{n^{\prime}}}{2}}\right)
\end{aligned}
$$

$\mathcal{E}$ is the amplitude of the radiation field; $\omega_{\mathrm{ab}}$ is an energy difference $: \hbar \omega_{\mathrm{ab}}=E_{\mathrm{a}}-E_{\mathrm{b}} ; \omega_{\mathrm{r}}$ is the laser frequency seen in the atom frame $; \Gamma_{n^{\prime}}$ is the inverse lifetime of the excited state. $\tau$ is the transition matrix which is connected to the interaction hamiltonian $\mathcal{H}^{\text {int }}$ between the atom and the electromagnetic field :

$$
\left\langle N-1 \mathbf{k}_{\mathrm{i}} \varepsilon\left|\otimes\left\langle n^{\prime} \mathrm{S}^{\prime} M^{\prime}\left|\mathcal{H}^{\mathrm{int}}\right| n S \varepsilon M\right\rangle \otimes\right| N \mathbf{k}_{\mathrm{i}} \varepsilon\right\rangle=\varepsilon\left\langle\varepsilon^{\prime} M^{\prime}|\tau| \varepsilon M\right\rangle
$$

( $\left.N \mathbf{k}_{\mathbf{i}} \varepsilon\right\rangle$ denoting a stationary state of the laser field).

$\tau$ can be decomposed into different transition amplitudes as discussed in previous papers $[1,8]$ :

$$
\tau=\alpha \mathbf{E}_{0} . \varepsilon+i \beta \sigma . \mathbf{E}_{0} \times \varepsilon-\left[M_{1}+\left(F^{\prime}-F\right) M_{1}^{\mathrm{hf}}\right] \sigma . \hat{k}_{\mathrm{i}} \times \varepsilon+i \operatorname{Im} E_{1}^{\mathrm{PV}} \boldsymbol{\sigma} . \varepsilon
$$

the components of $\sigma$ being the three Pauli matrices.

The first two terms constitute the Stark induced electric dipolar amplitude $E_{1}^{\text {ind }}, \alpha$ and $\beta$ being real coefficients. The ratio $\alpha / \beta$ has been determined experimentally for the $6 \mathrm{~S}-7 \mathrm{~S}$ Cs transition $[12,13]$. The last term is the parity violating electric dipolar amplitude. Its effects have been widely discussed elsewhere $[1,8]$. We are mainly interested here by manifestations of the magnetic dipolar amplitude (3rd term). Different small relativistic effects contribute to it; among them the combined effect of core polarization and spin-orbit interaction appears to be the dominant one for the 6 S-7 S Cs transition $[1,14]$. Next in importance comes the contribution $M_{1}^{\mathrm{hf}}$ of the hyperfine interaction which has non zero matrix elements between two states of different radial quantum numbers $n$ and different total angular momentum $F$. Thus the magnetic dipolar amplitude takes on different values : $M_{1}-M_{1}^{\text {hf }}, M_{1}$ and $M_{1}+M_{1}^{\text {hf }}$ for $\Delta F=-1,0$ and +1 components $\left(\Delta F=F^{\prime}-F\right)$. Presently, there are two experimental determinations of $M_{1} / \alpha$ agreeing with each other $[2,13]$; as to $M_{1}^{\mathrm{hf}}$, there is only one experi- 
mental determination of $M_{1}^{\mathrm{hf}} / M_{1}$ [13], which leads to a result larger than expected. So another measurement by an independent method, as the one proposed in section 4 , seems desirable. Indeed, the experimental value of $M_{1}^{\text {hf }}$ looks interesting for two reasons : first, it is a unique way of obtaining the off-diagonal matrix element of the hyperfine interaction; secondly, because it can be computed fairly accurately, it may be used in future experiments to calibrate other transition amplitudes, while, up to now, this role has been devoted to the Stark amplitude $\alpha E_{0}$.

In our calculation, we suppose the incident laser field quasi monochromatic $(\omega)$. For an experiment performed on atoms inside a vapour [2,13], we have to take into account the Boltzmann distribution of the absorbed frequencies; we perform the convolution product of $\rho_{n^{\prime}}\left(\omega_{r}\right)$ (given by eq. (3)) by the Doppler profile $\frac{1}{\sqrt{\pi} \Omega_{\mathrm{D}}} \exp -\left(\frac{\omega_{\mathrm{r}}-\omega}{\Omega_{\mathrm{D}}}\right)^{2}$.

We always consider the Doppler width as much larger than the natural Lorentzian width (for instance, at $T=472 \mathrm{~K}$, the former is $750 \mathrm{MHz}$ (FWHM) and the latter $3 \mathrm{MHz}$ ). As a consequence of the monochromaticity of the laser field, we have to consider the effect of the motional electric field $\mathbf{E}^{\text {mot }}$. As we shall see, a new interference mechanism then arises if $\mathbf{E}_{0}$ and $\mathbf{H}$ are crossed to the beam, since $\mathbf{E}^{\text {mot }}$ differs in general from zero even when averaging over the Boltzmann distribution. This new mechanism involves two Stark amplitudes, one induced by the external field $\mathbf{E}_{0}$, the other by the motional field.

Finally, let us note on equation (3) that the $\Gamma$-dependence disappears completely when dealing with populations $\left(\varepsilon_{1}^{\prime}=\varepsilon_{2}^{\prime}\right.$ and $\left.M_{1}^{\prime}=M_{2}^{\prime}\right)$ or with Zeeman coherences $\left(\varepsilon_{1}^{\prime}=\varepsilon_{2}^{\prime}=\varepsilon^{\prime}, M_{1}^{\prime} \neq M_{2}^{\prime}\right)$ in the limit of very weak magnetic fields $\left(\omega_{n^{\prime} \varepsilon^{\prime} M_{1}, n^{\prime} \varepsilon^{\prime} M_{2}} \ll \Gamma_{n^{\prime}}\right)$. As announced previously, these physical quantities transform under time reversal as if the excited state were a stable state.

\section{Simulation of $P V$ by a weak longitudinal magnetic} field. - We present in this section a perturbation calculation of PV simulation by a weak longitudinal magnetic field (a stray field) in the zero magnetic field experiment : such a field creates an electronic polarization normal to the beam and depending on the photon helicity. The field manifests itself in two different ways :

- it modifies the wavefunctions and thus the transition probabilities;

- it shifts the energy levels.

These two effects appear clearly in the calculation of $J P_{e}$ as seen from inspection of equations (2) and (3). The first order correction in low magnetic field results from two contributions. In the first one, all the energies are replaced by their zero $H$-field values and a first order correction is applied to the matrix elements. The frequency spectrum is composed of a set of Gaussian lines

$$
\mathfrak{G}_{F F^{\prime}}(\omega)=\exp -\frac{\left(\omega-\omega_{n^{\prime} F^{\prime} n F}\right)^{2}}{\Omega_{D}^{2}}
$$

centred at the different $F \rightarrow F^{\prime}$ transition frequencies, which result from the convolution product of Lorentz lines with the much wider Doppler profile. In the second contribution proportional to the energy level shift, the exact stationary states $|\varepsilon M\rangle$ are replaced by their zero field limit $|F M\rangle$. The associated frequency spectrum appears as a set of dispersive lines having the shape of the derivative of a Gaussian curve. For zero nuclear spin, only the second effect is present, since the energy levels are perturbed but not the eigenstates.

We shall not present here the details of the calculation which can be found in reference [15], but only the results concerning the effects which may cause systematic errors in the PV experiment in zero magnetic field. We shall express them in term of the genuine PV signal searched for in the fluorescence light intensity transmitted through a circular analyser $\left(\xi_{\mathrm{f}}\right)$, i.e. (up to an irrelevant arbitrary normalization factor) :

$\mathcal{S}_{F F^{\prime}}^{\mathrm{PV}}(\omega)=p_{F F^{\prime}} \mathfrak{G}_{F F^{\prime}}(\omega) \alpha E_{0} \operatorname{Im} E_{1}^{\mathrm{PV}}\left\{\xi_{\mathrm{i}} \xi_{\mathrm{f}} \hat{E}_{0} \times \hat{k}_{\mathrm{f}} \cdot \hat{k}_{\mathrm{i}}\right\}$,

where the pseudoscalar signature characteristic of a parity violating interaction appears clearly in the last factor inside brackets. For the $\boldsymbol{H}$-field contribution, written with the same normalization, we get :

$$
\begin{aligned}
\mathcal{S}_{F F^{\prime}}^{H}(\omega)=\left(a_{F F^{\prime}} \frac{\Omega_{\mathrm{D}}}{\omega^{*}}\right. & \left.+b_{F F^{\prime}} \frac{\omega-\omega_{n^{\prime} F^{\prime} n F}}{\Omega_{\mathrm{D}}}\right) \times \\
& \times \frac{\left|\mu_{\mathrm{B}}\right| \mathbf{H} \cdot \hat{k}_{\mathrm{i}}}{\hbar \Omega_{\mathrm{D}}} \frac{M_{1}}{\operatorname{Im} E_{1}^{\mathrm{PV}}} \mathcal{S}_{F F^{\prime}}^{\mathrm{PV}}(\omega)
\end{aligned}
$$

with the definition of the angular momentum factors $a_{F F^{\prime}}, b_{F F^{\prime}}, p_{F F^{\prime}}$ given in table I and :

$$
\begin{aligned}
\frac{1}{\omega^{*}} & =\frac{1}{\omega_{n}^{\mathrm{hf}}}\left(1+\frac{\beta}{\alpha} \frac{3+4 g_{F^{\prime}}}{5}\right)- \\
& \quad-\frac{1}{\omega_{n^{\prime}}^{\mathrm{hf}}}\left(1-\frac{\beta}{\alpha} \frac{1-12 g_{F^{\prime}}}{5}\right), \\
\Omega_{\mathrm{D}} & =\omega \sqrt{2 k T / M_{\mathrm{Cs}} c^{2}} \\
g_{F^{\prime}} & =2\left(F^{\prime}-I\right) /(2 I+1), \\
k_{F^{\prime}} & =\left(2 F^{\prime}+1\right)\left(\frac{1+2 g_{F^{\prime}}}{3}\right) .
\end{aligned}
$$

$\hbar \omega_{n}^{\mathrm{hf}}, \hbar \omega_{n^{\prime}}^{\mathrm{hf}}$ represent the hyperfine splittings of the ground and excited states, $\mu_{\mathbf{B}}$ is the Bohr magneton. 
Table I. - The angular momentum factors entering into the expressions of $\mathcal{S}^{H}(\omega)$ and $\mathcal{S}^{\mathrm{PV}}(\omega)$.

$$
\begin{array}{rrr} 
& F=F^{\prime} & F \neq F^{\prime} \\
& -\bar{\beta} & - \\
p_{F F^{\prime}} & -k_{F^{\prime}}\left(1+g_{F^{\prime}} \frac{\beta}{\alpha}\right) & -k_{F^{\prime}}\left(1-g_{F^{\prime}}\right) \frac{\beta}{\alpha} \\
b_{F F^{\prime}} & 2 g_{F^{\prime}} & \frac{2}{5}\left(2+g_{F^{\prime}}\right) \\
a_{F F^{\prime}} & 2\left(I-F^{\prime}\right)\left(1-g_{F^{\prime}}\right)\left(\left(1+g_{F^{\prime}} \frac{\beta}{\alpha}\right)\right. & 2\left(F^{\prime}-I\right) \frac{\alpha}{\beta}
\end{array}
$$

In figure 2, we have plotted, as a function of the incident light frequency $\omega$, the false signal $S^{H}(\omega)$ and the genuine one $S^{\mathrm{PV}}(\omega)$ for the $6 \mathrm{~S}-7 \mathrm{~S}$ cesium transition, supposing a half height Doppler width of $750 \mathrm{MHz}$. To conclude this section, we would like to make a few remarks :

1) The first concerns the order of magnitude of the systematic effect. Using the theoretical value of $\operatorname{Im} E_{1}^{\mathrm{PV}}$ for the $6 \mathrm{~S}-7 \mathrm{~S}$ transition computed in the framework of the Weinberg-Salam model [16] and the experimental value of $M_{1}$ [2] (leading to $\operatorname{Im} E_{1}^{\mathrm{PV}} / M_{1}=0.80 \times 10^{-4}$ ), we obtain for a laser frequency tuned near the $4 \rightarrow 4$ transition :

$$
\mathcal{S}^{H} / \mathcal{S}^{\mathrm{PV}}=\left(5.5+2.16 \times 10^{-2} \Delta v\right) H,
$$

$H$ being expressed in gauss and the detuning $\Delta v=\left(\omega-\omega_{4,4}\right) / 2 \pi$ in $\mathrm{MHz}$.

2) The effects associated respectively with the energy shifts and the perturbation of the eigenstates being of the same order of magnitude, it is possible by detuning the laser frequency by about $\pm 250 \mathrm{MHz}$ to cancel or double the systematic errors produced by a stray magnetic field.

3) The $\Delta F=0$ transitions are clearly favoured, the ratio $\mathcal{S}^{H} / \mathcal{S}^{\mathrm{PV}}$ being smaller for them than for $|\Delta F|=1$ by a factor $\alpha / \beta$, roughly equal to 10 .

4) Finally, it is very important to note the different behaviour of $S^{H}$ and $S^{P V}$ with respect to the reversal of the photon momentum $\mathbf{k}_{\mathbf{i}}$ : as long as the angular momentum $\xi_{\mathbf{i}} \mathbf{k}_{\mathbf{i}}$ stays unchanged (mirror reflexion at normal incidence), the reversal $\mathbf{k}_{\mathbf{i}} \rightarrow-\mathbf{k}_{\mathbf{i}}$ does

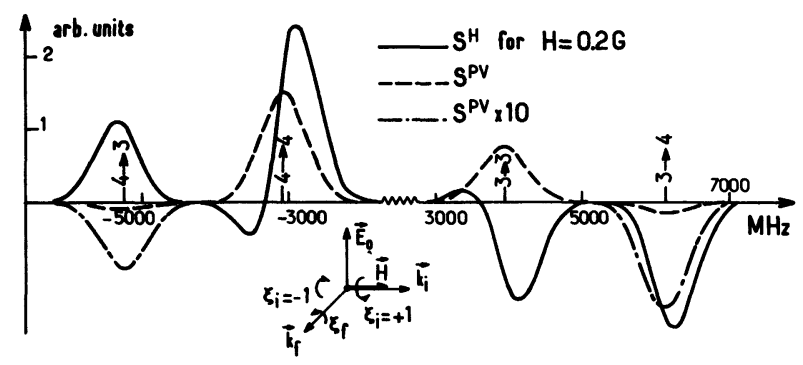

Fig. 2. - Frequency dependence of the PV signal, $\mathcal{S}^{\mathrm{PV}}$, and of the stray $\boldsymbol{H}$-field signal, $\mathcal{S}^{H}$, simulating it, for the cesium $6 \mathrm{~S}-7 \mathrm{~S}$ transition. The relative magnitude of $S^{\mathrm{PV}}$ and $S^{H}$ corresponds to a component of $H$ along the beam of $0.2 \mathrm{G}$, for a single pass of the laser beam. not affect $S^{P V}(5)$ whereas it changes $S^{H}$ into $-S^{H}$. So in practice, the $H$-field effect can be considerably reduced by sending the laser beam back on itself through the vapour.

4. $E_{1}^{\text {ind }}-M_{1}$ interference in the presence of transverse crossed electric and magnetic fields. - In the beginning of this paper, we have shown from simple symmetry considerations that a new kind of $M_{1}-E_{1}^{\text {ind }}$ interference effect could appear on the total fluorescence rate if a magnetic field was applied perpendicular to the beam and to $\mathbf{E}_{0}$. This is the effect that we are now going to analyse. We shall see that it suggests an interesting new possibility for measuring $M_{1}$ and $M_{1}^{\mathrm{hf}}$ provided that the magnetic field is relatively strong ( $\sim 1 \mathrm{kG})$.

We shall adopt here the geometrical configuration represented on figure $1 a$ with the incident linear polarization $\varepsilon$ parallel to $\mathbf{E}_{0}$ : this situation looks more interesting because the scalar amplitude $\alpha \mathbf{E}_{0} . \varepsilon$ is larger and simpler than the vectorial one $i \beta \mathbf{E}_{0} \times \boldsymbol{\sigma . \varepsilon}$ (for further details concerning the general case, see reference [15]).

We have performed a complete calculation for the $6 S_{1 / 2}-7 S_{1 / 2}$ cesium transition. We present here the results of the numerical computation performed (on UNIVAC 1110) as a function of the photon frequency for two values of the magnetic field (500 and $1000 \mathrm{G})$ for which the Zeeman splitting $(0.35 \mathrm{MHz}$ per gauss) is larger than the Doppler half height linewidth (750 $\mathrm{MHz}$ at $420 \mathrm{~K}$ ). To express the result, we choose the absorption cross section $\sigma_{\mathrm{a}}$ from which the fluorescence rate can be derived in any given experimental condition. If we neglect terms of the order of $\left(M_{1} / \alpha E_{0}\right)^{2}$, we can write $\sigma_{\mathrm{a}}$ as :

$$
\begin{aligned}
& \sigma_{\mathrm{a}}(H, \omega)=\bar{\sigma}_{\mathrm{a}}\left[u_{\alpha}(H, \omega)-2\left(v_{\alpha}(H, \omega) \frac{M_{1}}{\alpha E_{0}}+\right.\right. \\
& \left.\left.+w_{\alpha}(H, \omega) \frac{M_{1}^{\mathrm{hf}}}{\alpha E_{0}}+t_{\alpha}(H, \omega) \frac{H_{\mathrm{c}}}{E_{0}} \frac{\Omega_{\mathrm{D}}}{\omega}\right) \hat{k}_{\mathrm{i}} \times \hat{E}_{0} \cdot \hat{H}\right]
\end{aligned}
$$

$\bar{\sigma}_{\mathrm{a}}$ is the resonance cross-section in the zero $\boldsymbol{H}$-field limit summed over the two $\Delta F=0$ transitions, proportional to $\alpha^{2} E_{0}^{2}$. Quantities $u_{\alpha}, v_{\alpha}, w_{\alpha}$ and $t_{\alpha}$ are dimensionless and function of the magnetic field $H$ and of the laser frequency $\omega\left(^{2}\right)$. They are plotted versus $\omega$ for two field magnitudes $H=500 \mathrm{G}$ (Fig. 3) and $H=1000 \mathrm{G}$ (Fig. 4). $u_{\alpha}$ is associated with the quadratic term $\left|E_{1}^{\text {ind }}\right|^{2} ; v_{\alpha}$ and $w_{\alpha}$ correspond to the interference terms $E_{1}^{\text {ind }} M_{1}$ and $E_{1}^{\text {ind }} M_{1}^{\text {hf }} ; t_{\alpha}$ describes the interference between the electric dipole amplitudes induced by the applied and the motional Stark fields $E_{0} E^{\text {mot: }}$ As already mentioned, the motional electric field effect results from the monochromaticity

(2) With another orientation of $\varepsilon$ with respect to $\mathbf{E}_{0}$ the polarizability $\beta$ also contributes and we introduce four other quantities $u_{\beta}, v_{\beta}, w_{\beta}, t_{\beta}$ [15]. 

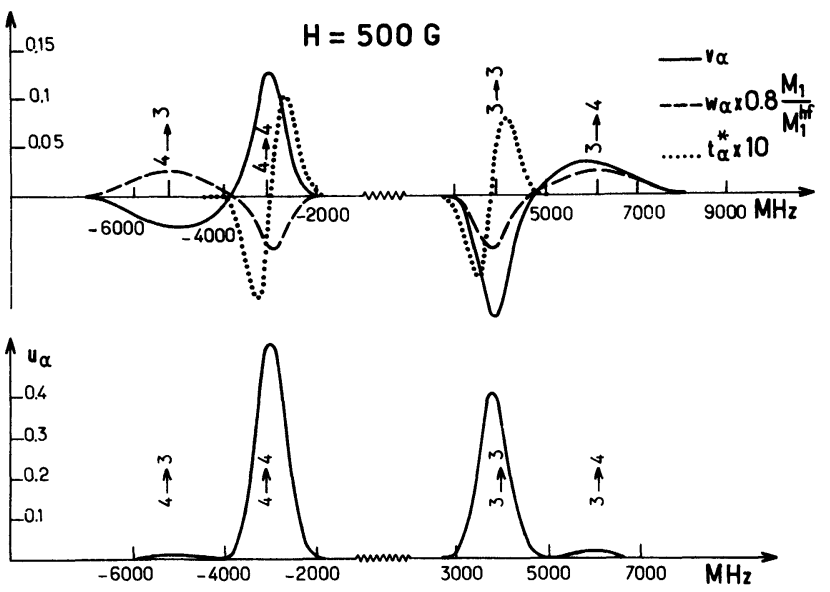

Fig. 3.

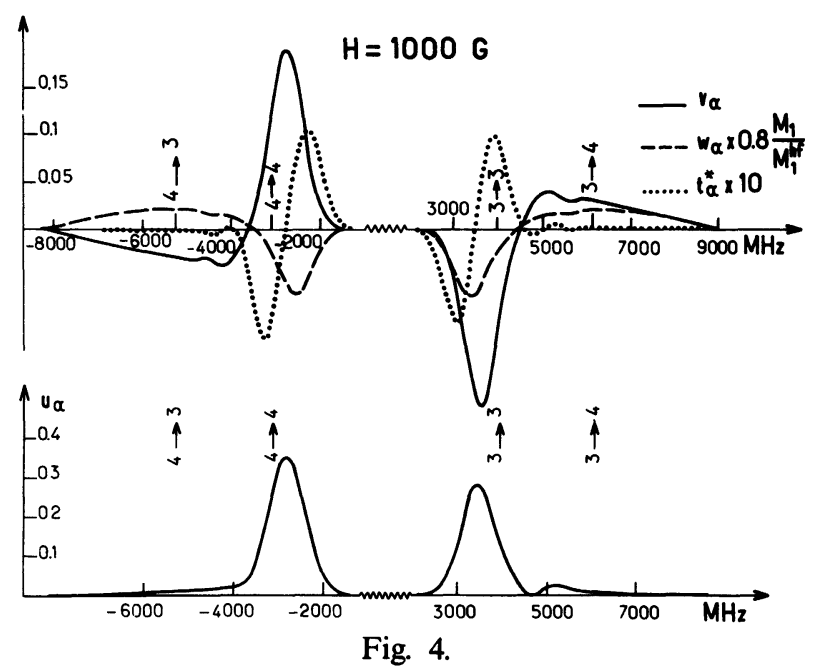

Figs. 3 and 4. - Absorption cross-section for the cesium $6 \mathrm{~S}_{1 / 2}-7 \mathrm{~S}_{1 / 2}$ transition versus laser frequency, in crossed electric and magnetic fields with a beam linearly polarized parallel to $\mathbf{E}_{0}$. Figures 3 and 4 are relative to $H=500 \mathrm{G}$ and $H=1000 \mathrm{G}$ respectively

$$
\sigma_{\mathrm{a}}=\bar{\sigma}_{\mathrm{a}}\left[u_{\alpha}-2\left(v_{\alpha} \frac{M_{1}}{\alpha E_{0}}+w_{\alpha} \frac{M_{1}^{\mathrm{hf}}}{\alpha E_{0}}+t_{\alpha} \frac{H c}{E_{0}} \frac{\Omega_{\mathrm{D}}}{\omega}\right) \hat{k}_{\mathrm{i}} \times \hat{E}_{0} . \hat{H}\right]
$$

The lower curve represents the main contribution $u_{\alpha}$. Upper curves give the contributions $v_{\alpha}$ with the same scale, $w_{\alpha}$ with a scale expanded by the ratio $0.8 \frac{M_{1}}{M_{1}^{\mathrm{hf}}}$ and

$$
t_{\alpha}^{*}=t_{\alpha} \frac{\alpha H_{c}}{M_{1}} \frac{\Omega_{\mathrm{D}}}{\omega}
$$

with a scale expanded by 10 . We use the value $M_{1} / \alpha=2.62 \mathrm{~V} / \mathrm{cm}$ from reference [2]. The zero field hyperfine components $(3 \rightarrow 3,3 \rightarrow 4$, etc.) are indicated. Zero frequency is the transition frequency without hfs.

of the laser excitation : for some atoms having the right velocity along the beam $\left(v \cdot \hat{k}_{\mathrm{i}}\right)$, the laser frequency in the rest frame coincides with one of the atomic frequencies. These atoms are excited with a stronger probability and consequently the average value of $\mathbf{v} . \hat{k}_{\mathrm{i}}$ taken over the excited state population is non zero (on the opposite, the velocity components transverse to the beam of course average to zero).
The signature of the $E_{0} E^{\text {mot }}$ interference effect is thus easy to understand :

$$
\mathbf{E}_{0} \mathbf{E}^{\mathrm{mot}}=\left\langle\mathbf{v} . \hat{k}_{\mathrm{i}}\right\rangle \hat{k}_{\mathrm{i}} \times \mathbf{H} . \mathbf{E}_{\mathbf{0}} .
$$

As announced, the motional effect has the same signature than the $M_{1} E_{1}^{\text {ind }}$ effect. Noting now that the atom velocity v. $\hat{k}_{\mathrm{i}}$ determined by the Doppler effect can take different possible values :

$$
v=\frac{\omega-\omega_{n^{\prime} \varepsilon^{\prime} M, n \varepsilon M}}{\omega} c
$$

and using the definitions of $u_{\alpha}$ and $t_{\alpha}$, we obtain a very simple relation :

$$
t_{\alpha}=-\frac{\Omega_{\mathrm{D}}}{2} \frac{\mathrm{d} u_{\alpha}}{\mathrm{d} \omega} .
$$

This explains why the zeros of $t_{\alpha}$ occur for the maxima of $u_{\alpha}$ (Figs. 3 and 4). The order of magnitude of the amplitude $\alpha E^{\text {mot }}$ must be compared to $M_{1}$, we get :

$$
\frac{\alpha E^{\mathrm{mot}}}{M_{1}} \simeq \frac{\alpha H c}{M_{1}} \frac{\Omega_{\mathrm{D}}}{\omega}=0.93 \times 10^{-4} \times H \text { (gauss) } .
$$

In a field of $1000 \mathrm{G}$ which has the right order of magnitude to make the $\alpha E_{0} M_{1}$ interference fully effective (as can be seen on figure 4, $2 v_{\alpha}$ becomes of the order of $u_{\alpha}$ ), the ratio $\alpha E^{\mathrm{mot}} / M_{1}$ is found to be 0.1 , so we see that the motional field cannot be neglected. This is confirmed by figures 3 and 4 where we plot with an expanded scale the quantity

$$
t_{\alpha}^{*}=t_{\alpha} \frac{\alpha H c}{M_{1}} \frac{\Omega_{\mathrm{D}}}{\omega}
$$

to be compared to $v_{\alpha}$.

Let us now indicate by which mechanism the new $\alpha E_{0} M_{1}$ and $\alpha E_{0} M_{1}^{\mathrm{hf}}$ interference effects appear : this is particularly simple for $M_{1}^{\text {hf }}$. In zero $H$-field, the $\alpha E_{0} M_{1}^{\text {hf }}$ interference cannot exist on $\Delta F=0$ transitions since $M_{1}^{\text {hf }}$ has no matrix element and it cannot either on $|\Delta F|=1$ transitions for which the scalar amplitude has zero element. So the important role played by the magnetic field comes from the modification of the eigenstates : when $F$ is no longer a good quantum number, both amplitudes become simultaneously present and can interfere. Concerning the $\alpha E_{0} M_{1}$ interference, we note that non zero $\alpha E_{0}$ matrix elements exist only for $\Delta F=0, \Delta M_{F}=0$ transitions which are unaffected by the level shift mechanism alone. Consequently, as for the $\alpha E_{0} M_{1}^{\text {hf }}$ interference, the dominant role is played by the eigenstate perturbation mechanism. For this reason, it is not surprising to note on figures 3 and 4 that near the zero-field transition frequencies where $t_{\alpha}$ cancels out, we observe only slow variations of $v_{\alpha}$ and $w_{\alpha}$.

There is now another very interesting feature quite apparent on figures 3 and 4 : the curve $v_{\alpha}$ is odd and 
the curve $w_{\alpha}$ is even with respect to the central frequency $\omega_{\mathrm{c}}=\left(\omega_{n}^{\mathrm{hf}}-\omega_{n^{\prime}}^{\mathrm{hf}}\right) / 2(2 I+1)=439 \mathrm{MHz}$ (this frequency is equidistant from the $3 \rightarrow 3$ and $4 \rightarrow 4$ zero-field transition frequencies and equidistant also from the $3 \rightarrow 4,4 \rightarrow 3$ ). Let us indicate here that this symmetry is actually rigorous and can be demonstrated by simple considerations of angular momentum algebra. In an experimental determination of the ratio $M_{1}^{\mathrm{hf}} / M_{1}$, one can take advantage of this property : if the fluorescence rate $\mathcal{F}$, linear in $E_{0}$, is measured at 2 frequencies symmetric with respect to $\omega_{c}$, giving results $\mathcal{F}_{1}$ and $\mathcal{F}_{2}$, we see that the ratio $\left(\mathcal{F}_{1}+\mathcal{F}_{2}\right) /\left(\mathcal{F}_{1}-\mathcal{F}_{2}\right)$ is directly connected to $M_{1}^{\mathrm{hf}} / M_{1}$. However some complication occurs because of the $E_{1}^{\text {ind }} E^{\text {mot }}$ term which exhibits no particular symmetry when varying the laser frequency. To get round this difficulty, it is possible to choose frequencies where the $E^{\text {mot }}$ effect is negligible : this is the case when $u_{\alpha}$ is very small ( $\Delta F= \pm 1$ components) or when $u_{\alpha}$ is extremal $\left(\right.$ then $\left.t_{\alpha} \propto \frac{\mathrm{d} u_{\alpha}}{\mathrm{d} \omega}=0\right)$.

To conclude this section, we propose a method for measuring $M_{1}^{\mathrm{hf}} / M_{1}$ which brings several improvements with respect to the zero-field experiments : polarization detection becoming unnecessary, the efficiency of fluorescence light collection can be increased; this improves the signal to noise ratio; depolarization effects become unimportant and calibration can be performed in different ways either by using the dominant contribution or by comparison with the motional effect which can be extracted from the fluorescence rate linear in $E_{0}$.

\section{APPENDIX}

We present here a derivation of formula (3) for the density matrix elements in the excited state. The optical excitation, resonant for the $n S \rightarrow n^{\prime} \mathrm{S}$ transition, can only populate the $n^{\prime} \mathrm{S}$ level.

Let $\rho$ be the density matrix of the system atom plus laser field, $\Lambda_{n}$ and $\Lambda_{n}$, the projectors on the states

$$
\left|n S_{1 / 2}\right\rangle \otimes\left|N k_{i} \varepsilon\right\rangle \text { and }\left|n^{\prime} S_{1 / 2}\right\rangle \otimes\left|N-1 \mathbf{k}_{\mathrm{i}} \varepsilon\right\rangle \text {. }
$$

$N$ denotes the number of incident photons of frequency $\omega$, momentum $\mathbf{k}_{\mathbf{i}}$, polarization $\varepsilon$, inside the quantization box of volume $V$. We define :

$$
\begin{aligned}
\rho_{n} & =\Lambda_{n} \rho \Lambda_{n} & \rho_{n^{\prime} n} & =\Lambda_{n^{\prime}} \rho \Lambda_{n} \\
\rho_{n n^{\prime}} & =\Lambda_{n} \rho \Lambda_{n^{\prime}} & \rho_{n^{\prime}} & =\Lambda_{n^{\prime}} \rho \Lambda_{n^{\prime}}
\end{aligned}
$$

and we get a reduced density matrix :

$$
\rho_{\mathrm{red}}=\left(\begin{array}{ll}
\rho_{n} & \rho_{n n^{\prime}} \\
\rho_{n^{\prime} n} & \rho_{n^{\prime}}
\end{array}\right)
$$

In the same way, we define a reduced hamiltonian by the following relation :

$$
\Lambda_{n} \mathfrak{H} \Lambda_{n}=\mathcal{H}_{n}^{\text {at }}, \quad \Lambda_{n^{\prime}} \mathcal{H} \Lambda_{n^{\prime}}=\mathcal{H}_{n^{\prime}}^{\text {at }}+\hbar\left(\omega_{n^{\prime} n}-\omega_{\mathrm{r}}\right)
$$

$\omega_{n^{\prime} n}$ being the transition energy between the barycentres of the $n^{\prime} S$ and $n S$ levels and $\omega_{\mathrm{r}}$ the laser frequency seen in the atom frame. The atom-laser field interaction is written :

$$
\mathcal{H}^{\text {int }}=-\mathbf{d} . \boldsymbol{\delta}-\boldsymbol{\mu} . \mathscr{H}
$$

d and $\boldsymbol{\mu}$ being the electric and magnetic dipoles of the atom, $\boldsymbol{E}$ and $\mathfrak{X}$ the electric and magnetic fields of the incident light wave. So the non-diagonal terms $\mathscr{H}_{n n^{\prime}}$ involve the laser field (we replace its quantized expression $\sqrt{\frac{2 \pi N \hbar \omega}{V}}$ by $\left.\mathcal{E}\right)$ and the transition matrix $\tau$ already defined in section 3 , equation (4) :

$$
\Lambda_{n^{\prime}} \mathfrak{H} \Lambda_{n}=\delta \tau
$$

The $n S \rightarrow n^{\prime} \mathbf{S}$ transition is so highly forbidden that stimulated emission is negligible with respect to spontaneous emission $\left(n^{\prime} \mathrm{S} \rightarrow n^{\prime \prime} \mathrm{P}\right)$. The spontaneous decay is isotropic and can be taken into account by the following term [17] :

$$
\left.\frac{\partial}{\partial t} \rho_{\text {red }}\right|_{\substack{\text { spontaneous } \\
\text { decay }}}=-\Gamma\left(\rho_{\text {red }}\right)=-\left(\begin{array}{cc}
0 & \frac{\Gamma_{n^{\prime}}}{2} \rho_{n n^{\prime}} \\
\frac{\Gamma_{n^{\prime}}}{2} \rho_{n^{\prime} n} & \Gamma_{n^{\prime}} \rho_{n^{\prime}}
\end{array}\right) .
$$


Therefore we write the Schrödinger equation :

$$
\frac{\partial}{\partial t} \rho_{\text {red }}=\frac{1}{i \hbar}\left[\mathcal{H}_{\text {red }}, \rho_{\text {red }}\right]-\Gamma\left(\rho_{\text {red }}\right) .
$$

We simply solve this equation by a perturbative method : the transition is not saturated because it is highly forbidden. At zero order in laser intensity, the density matrix is assumed to be ruled by thermal equilibrium :

$$
\rho_{\text {red }}=\left(\begin{array}{cc}
\frac{1}{2(2 I+1)} & 0 \\
0 & 0
\end{array}\right) \text {. }
$$

We deduce from (9) the evolution of an optical coherence :

$$
\frac{\partial}{\partial t} \rho_{n n^{\prime}}=\frac{1}{i \hbar}\left[\mathscr{H}_{n}^{\mathrm{at}} \rho_{n n^{\prime}}-\rho_{n n^{\prime}}\left(\mathscr{H}_{n^{\prime}}^{\mathrm{at}}+\hbar\left(\omega_{n^{\prime} n}-\omega_{\mathrm{r}}+i \frac{\Gamma_{n^{\prime}}}{2}\right)\right)+\mathcal{E}\left(\tau^{+} \rho_{n^{\prime}}-\rho_{n} \tau\right)\right] .
$$

We restrict ourselves to stationary solutions, noted $\rho^{\mathbf{s}}$. By inserting (10) into (11) and writing $\frac{\partial}{\partial t}\left\langle\varepsilon M\left|\rho_{n n^{\prime}}^{\mathrm{s}}\right| \varepsilon_{2}^{\prime} M_{2}^{\prime}\right\rangle=0$, we obtain :

$$
\left\langle\varepsilon M\left|\rho_{n n^{\prime}}^{\mathrm{S}}\right| \varepsilon_{2}^{\prime} M_{2}^{\prime}\right\rangle=\frac{\left\langle\varepsilon M\left|\delta \tau^{+}\right| \varepsilon_{2}^{\prime} M_{2}^{\prime}\right\rangle}{2(2 I+1) \hbar\left(\omega_{\mathrm{r}}-\omega_{n^{\prime} \varepsilon_{2}^{\prime} M_{2}^{\prime}, n \varepsilon M}-i \frac{\Gamma_{n^{\prime}}}{2}\right)} .
$$

We now write the Schrödinger equation for $\rho_{n^{\prime}}$, derived from (9) :

$$
\frac{\partial}{\partial t} \rho_{n^{\prime}}=\frac{1}{i \hbar}\left[\left[\mathcal{H} \Theta_{n^{\prime}}^{\mathrm{at}}, \rho_{n^{\prime}}\right]+\delta\left(\tau \rho_{n n^{\prime}}-\rho_{n n^{\prime}} \tau^{+}\right)\right]-\Gamma_{n^{\prime}} \rho_{n^{\prime}} .
$$

We derive from (12) and (13) the stationary component of $\rho_{n^{\prime}}$.

$$
\begin{aligned}
& \left\langle\varepsilon_{1}^{\prime} M_{1}^{\prime}\left|\rho_{n^{\prime}}^{\mathrm{S}}\right| \varepsilon_{2}^{\prime} M_{2}^{\prime}\right\rangle=\frac{\varepsilon^{2}}{2(2 I+1) \hbar^{2}}\left(\omega_{n^{\prime} \varepsilon_{1}^{\prime} M_{1}^{\prime}, n^{\prime} \varepsilon_{2}^{\prime} M_{2}^{\prime}}-i \Gamma_{n^{\prime}}\right)^{-1} \times \\
& \quad \times \sum_{\varepsilon M}\left\langle\varepsilon_{1}^{\prime} M_{1}^{\prime}|\tau| \varepsilon M\right\rangle\left\langle\varepsilon M\left|\tau^{+}\right| \varepsilon_{2}^{\prime} M_{2}^{\prime}\right\rangle\left(\frac{1}{\omega_{\mathrm{r}}-\omega_{n^{\prime} \varepsilon_{1}^{\prime} M_{1}^{\prime}, n \varepsilon M}+i \frac{\Gamma_{n^{\prime}}}{2}}-\frac{1}{\omega_{\mathrm{r}}-\omega_{n^{\prime} \varepsilon_{2}^{\prime} M_{2}, n \varepsilon M}-i \frac{\Gamma_{n^{\prime}}}{2}}\right)
\end{aligned}
$$

$\operatorname{Tr} \rho_{n^{\prime}}^{\mathbf{S}}$ represents the absorption cross-section at frequency $\omega$ in a stationary regime, and $\operatorname{Tr} \rho_{n^{\prime}}^{\mathbf{S}} \boldsymbol{\sigma} / \operatorname{Tr} \rho_{n^{\prime}}^{\mathbf{S}}, \bar{t}$ the stationary component of the atomic orientation $\mathbf{P}_{\mathrm{e}}$.

\section{References}

[1] Bouchiat, M. A. and Bouchiat, C., J. Physique 36 (1975) 493.

[2] Bouchiat, M. A. and Pottier, L., J. Physique Lett. 37 (1976) L-79.

[3] Chu, S., Commins, E. D. and Conti, R., Phys. Lett. 60A (1977) 96.

[4] Itano, W. M., Phys. Rev. A 22 (1980) 1558.

[5] Drake, G. W. F., Phys. Rev. Lett. 40 (1978) 1705.

[6] Bouchiat, M. A. and Pottier, L., Phys. Lett. 62B (1976) 327 and Proc. Int. Workshop on Neutral Current Interactions in Atoms, Cargese, 1979 (W. L. Williams, ed.), p. 122.

[7] Conti, R., Bucksbaum, P., Chu, S., Commins, E. and Hunter, L., Phys. Rev. Lett. 42 (1979) 343

[8] Bouchiat, M. A., Poirier, M. and Bouchiat, C., $J$. Physique 40 (1979) 1127.

[9] Bucksbaum, P., Proc. Int. Workshop on Neutral Current Interactions in Atoms, Cargese, 1979 (W. L. Williams, ed.), p. 92.
[10] Bell, J. S., Proc. Int. Workshop on Neutral Current Interactions in Atoms, Cargese, 1979 (W. L. Williams, ed.), p. 288.

[11] Dunford, R. W., Lewis, R. R., Williams, W. L., Phys. Rev. A 18 (1978) 2421.

[12] Bouchiat, M. A. and Pottier, L., J. Physique Lett. 36 (1975) L-189.

[13] Hoffnagle, J., Roesch, L. P., Telegdi, V. L., Weis, A. and ZeHNDER, Phys. Lett. 85A (1981) 143.

[14] Flanbaum, V. V., Khriplovich, I. B. and Sushkov, O. P., Phys. Lett. 67A (1978) 177.

[15] PoIrIER, M., Thèse de 3e cycle, Paris VI University (1979), unpublished.

[16] Bouchiat, C., Atomic Physics 7, edited by D. Kleppner and F. Pipkin (Plenum Press) 1981, p. 83.

[17] Mollow, B. R. and Miller, M. M., Ann. Phys. 52 (1969) 464. 